\title{
Nanotheranostics
}

2017; 1(2): 196-207. doi: 10.7150/ntno.18576

Research Paper

\section{Doxorubicin/Ce6-Loaded Nanoparticle Coated with Polymer via Singlet Oxygen-Sensitive Linker for Photodynamically Assisted Chemotherapy}

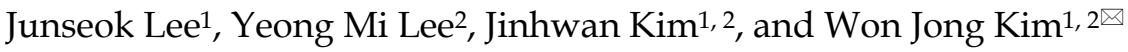 \\ 1. Center for Self-assembly and Complexity, Institute of Basic Science (IBS), Pohang, 37673, Korea; \\ 2. Department of Chemistry, Pohang University of Science and Technology (POSTECH), Pohang, 37673, Korea. \\ $\triangle$ Corresponding author: E-mail: wjkim@postech.ac.kr Fax: +82-54-279-3399 \\ (C) Ivyspring International Publisher. This is an open access article distributed under the terms of the Creative Commons Attribution (CC BY-NC) license \\ (https://creativecommons.org/licenses/by-nc/4.0/). See http://ivyspring.com/terms for full terms and conditions.
}

Received: 2016.12.01; Accepted: 2017.02.19; Published: 2017.05.08

\begin{abstract}
It is widely known that the therapeutic effect of nanoparticle-based chemotherapeutics could be greatly enhanced by the introduction of the photodynamic effect. Herein we report a chlorin e6-incorporated mesoporous silica nanoparticles (MSNs) covered with a polyethylene glycol shell conjugated via a singlet oxygen-sensitive labile bis(alkylthio)alkene linker (CeAP-L-PEG). In this study, single irradiation with biocompatible red light induced both intracellular doxorubicin release and photochemical internalization, and consequently enhanced anti-cancer effect was observed in vitro and in vivo. This study suggests the potential of our precisely designed nanoparticle system for photodynamically assisted chemotherapy.
\end{abstract}

Key words: Mesoporous silica, Singlet oxygen, Photodynamic therapy, Chemotherapy, Photochemical internalization.

\section{Introduction}

Chemotherapy is one of the most popular therapeutic methods for the treatment of various types of cancers. In many cases, however, drug resistance occurs during therapy and makes the disease inextirpable.1, 2 Recently, the combination of chemotherapy with other strategies has attracted attention as it can overcome limits of conventional single treatment by using different modes of action. For instance, photothermal therapy could be an appropriate option to enhance therapeutic effects because tumor cells are vulnerable to thermal damage. ${ }^{3-5}$ Another suitable strategy is photodynamic therapy. ${ }^{6}$ According to several reports, the anti-cancer effects of chemotherapeutics such as doxorubicin were remarkably enhanced when chemotherapy was combined with photodynamic therapy (PDT). ${ }^{7,8}$

PDT is a light-mediated therapeutic method based on the use of photosensitizers (PS) under irradiation with an appropriate wavelength of light. ${ }^{9}$ It is widely used in the medical field for the treatment of not only cancer but also dermatological or ophthalmic diseases. ${ }^{10}$ Under irradiation, the PS is photo-activated and reactive oxygen species (ROS), including free radicals and singlet oxygen $\left({ }^{1} \mathrm{O}_{2}\right)$, are generated. As-generated ${ }^{1} \mathrm{O}_{2}$ is a highly reactive molecule and thus, it readily reacts with nearby biomolecules including phospholipids or DNA, which further leads to apoptosis or necrosis of the targeted tissue. ${ }^{11}$ Moreover, severe oxygen depletion occurs when a large amount of oxygen is activated by PS.12 Although PDT is a powerful method for cancer therapy by itself, the inherent properties of PS molecules, such as poor solubility and self-quenching effects, remain as obstacles to be overcome. To date, numerous biocompatible vehicles including liposomes, polymeric micelles, self-assembled polymeric nanoparticles, and solid nanoparticles such as gold or silica have been developed to increase the therapeutic efficiency of PDT. ${ }^{13-23}$ However, non-specific release of loaded PS through the 
destruction of the carrier would give rise to diminishing efficiency and may cause severe side effects. Unlike conventional chemotherapy, the release of the loaded PS is not a prerequisite for PDT since the molecule in action is not the PS itself. Thus, the introduction of PS as a conjugated form into a solid nanoparticle would be an alternative for the successful delivery of PS. 18,24

Interestingly, it has been reported that the photodynamic effect can enhance the therapeutic effect of nanoparticle-mediated chemotherapy. After entering a cell by endosomal uptake, when the nanoparticles are irradiated with light to induce the photodynamic effect, the endosomal membrane is oxidized by as-generated ROS and further disrupted, a phenomenon known as photochemical internalization (PCI). ${ }^{25}, 26$ Thus, since the delivered drug is easily degraded in the endosomal environment, it is possible to take advantage of PCI to assist with the successful intracellular release of delivered chemotherapeutics. In that point of view, the combination of PS and an ${ }^{1} \mathrm{O}_{2}$-cleavable linker could be good strategy to render a photodynamically controllable drug delivery platform. ${ }^{27}$ Under a single irradiation with light, ${ }^{1} \mathrm{O}_{2}$ is generated, which can not only trigger drug release but also facilitate endosomal escape. Moreover, a dual effect of PDT by ${ }^{1} \mathrm{O}_{2}$ and chemotherapy by released chemotherapeutics can be expected as aforementioned. Therefore, the development of a photodynamically controlled drug delivery system would be promising in an advanced photo-responsive platform.

Previously, our group has reported a drug release system that is responsive to long-wavelength light based on mesoporous silica nanoparticles (MSNs). ${ }^{28}$ In this system, a PS responsive to long-wavelength light was loaded into the porous structure of MSNs, and a model fluorescent dye was conjugated on the surface via a singlet oxygen-sensitive linker (SOSL). Irradiation with long-wavelength light generated ${ }^{1} \mathrm{O}_{2}$ and readily broke the SOSL, triggering the highly sensitive release of the model drug from the surface.

Inspired by previous reports by Singh et al. and our group, we further developed these studies by designing an MSN-based drug release system with an ${ }^{1} \mathrm{O}_{2}$-responsive PEG shell on the surface. ${ }^{21,}{ }^{29}$ Scheme 1 illustrates the photo-responsive drug release system consisting of MSNs with chlorin e6 (Ce6) conjugated in a porous structure and PEG conjugated by a SOSL on the surface of the MSNs. Similar to the results of a previous study, ${ }^{1} \mathrm{O}_{2}$ was generated upon irradiation with red light $(660 \mathrm{~nm})$ and was utilized for both photodynamically triggered drug release and photodynamic therapy. Furthermore, ${ }^{1} \mathrm{O}_{2}$ mediated the endosomal escape of the nanoparticles, and drug release were expected. Since a single stimulation could induce photodynamic therapy and chemotherapy simultaneously the potential for enhanced cancer therapy was evaluated in vitro and in vivo.
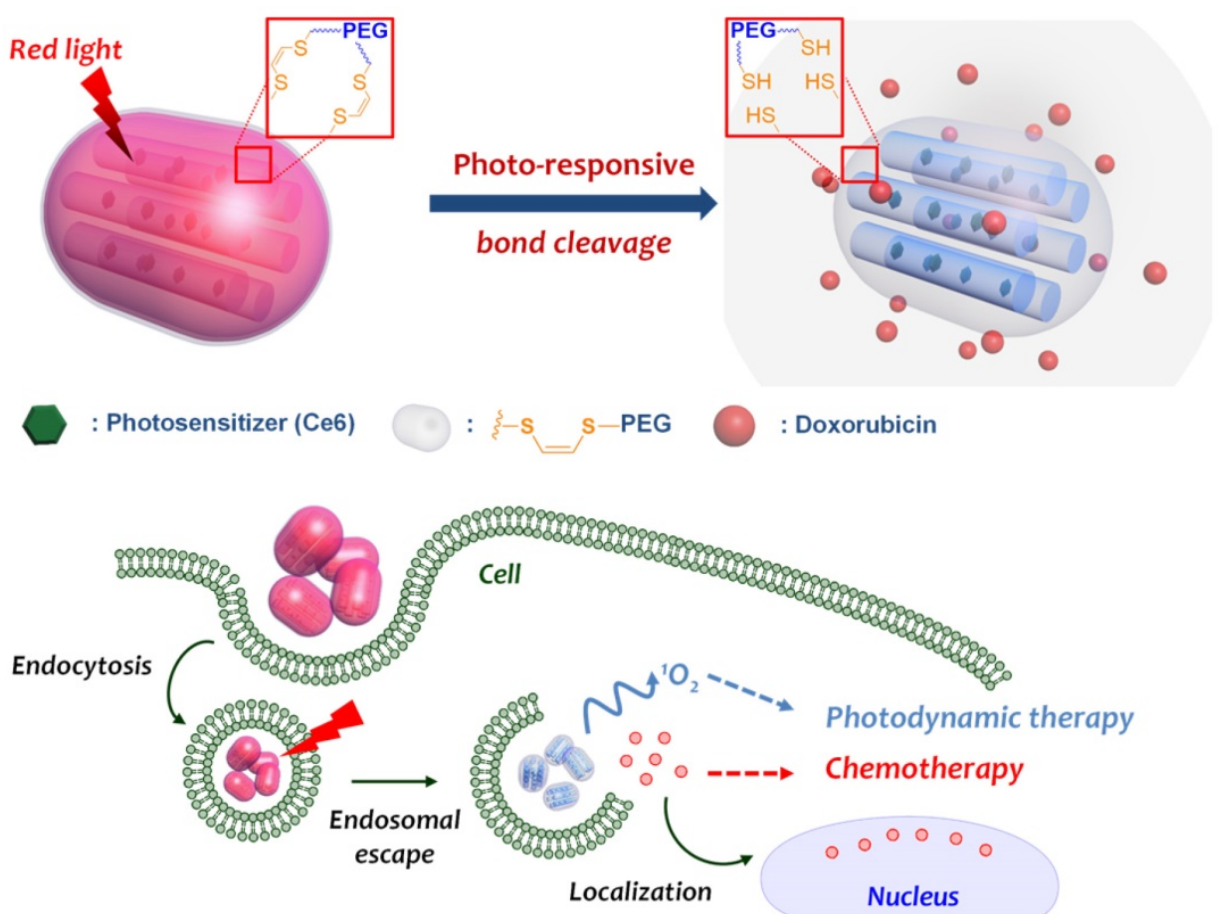

Scheme 1. Schematic illustration of photo-responsive drug releasing system triggered by as-generated singlet oxygen from the photo-activation of photosensitizer under irradiation with red light, and intracellular behavior of delivered nanoparticle. 


\section{Materials and methods}

\section{Reagents}

Cetyltrimethylammonium bromide (CTAB), sodium hydroxide $(\mathrm{NaOH})$, tetraethyl orthosilicate (TEOS), (3-aminopropyl)triethyoxysilane (APTES), 3-mercaptopropionic acid (3-MPA), cis-1,2-dichloroethylene, fluorescamine, 1-ethyl-3-(3-dimethylaminopropyl) carbodiimide hydrochloride (EDC-HCl), N-hydroxysuccinimide (NHS), poly(ethyleneglycol) diacrylate (PEGDA; $\mathrm{MW}=700$ ), ammonium persulfate (APS), $N, N, N^{\prime}, N^{\prime}$-Tetramethylethylenediamine (TEMED) and thiazolyl blue tetrazolium bromide (MTT) were purchased from Sigma Aldrich. 30 wt \% $\mathrm{NaOMe}$ in $\mathrm{MeOH}$ was purchased from Acros. Singlet oxygen sensor green ${ }^{\circledR}$ (SOSG) reagent was purchased from Molecular Probes (Eugene, OR). Doxorubicin hydrochloride (DOX) was purchased from Wako Chemical (Japan). Chlorin e6 (Ce6) was purchased from Frontier Scientific (Logan, UT). All reagents were used as received without further purification. DMF was dried under $\mathrm{CaH}_{2}$ and distilled.

\section{Instrumental methods}

TEM image was taken from a transmission electron microscope (JEM-2210, JEOL) and analysed by Gatan DigitalMicrograph software. UV-vis spectra were measured from a UV-vis spectrometer (UV 2550, Shimadzu) and fluorescence spectra were measured from a spectrofluorophotometer (RF-5301 PC, Shimadzu). Hydrodynamic volume was measured from zetasizer (Nano S90, Malvern) at $0.1 \mathrm{mg} / \mathrm{mL}$ in phosphate buffered saline (PBS) and zeta potential was measured from zetasizer (Nano Z, Malvern) at 0.1 $\mathrm{mg} / \mathrm{mL}$ in PBS. Confocal laser scanning microscope (CLSM) image was obtained from Olympus FV-1000 and analysed by OLYMPUS FLUOVIEW ver. 1.5 Viewer software.

\section{Preparation of photosensitizer conjugated organosilane (Ce6-TES)}

Ce6-TES was prepared by amide coupling reaction between $\mathrm{COOH}$ of $\mathrm{Ce} 6$ and $\mathrm{NH}_{2}$ of APTES (Scheme S1). ${ }^{1}$ Briefly, $105 \mathrm{mg}$ of Ce6 $(0.177 \mathrm{mmol})$ was dissolved in $10 \mathrm{~mL}$ of dry DMF. $170 \mathrm{mg}$ of EDC-HCl and $115 \mathrm{mg}$ of NHS were added into the solution (both $5 \mathrm{eq}$ ) and $207 \mu \mathrm{L}$ of APTES (5 eq) was added after $15 \mathrm{~min}$ of activation. The solution was stirred at RT for overnight. The final product was introduced for the synthesis of mesoporous silica without further purification.
Preparation of Ce6-conjugated and amine-modified mesoporous silica (CeAP)

Surface amine-modified and pore Ce6-conjugated mesoporous silica (CeAP) was prepared by the reported method with some modification. ${ }^{2} 200 \mathrm{mg}$ of CTAB was dispersed in 96 $\mathrm{mL}$ of distilled water (DW) and $700 \mu \mathrm{L}$ of $2 \mathrm{M} \mathrm{NaOH}$ solution was added. The suspension was stirred at 80 ${ }^{\circ} \mathrm{C}$ for $30 \mathrm{~min}$ and $1 \mathrm{~mL}$ of TEOS and $1 \mathrm{~mL}$ of as-prepared Ce6-TES solution in DMF were added. $117 \mu \mathrm{L}$ of APTES was added after 15 min and further stirred for $2 \mathrm{~h}$. Reaction mixture was centrifuged $(13,000 \mathrm{rpm}, 30 \mathrm{~min})$ to isolate the nanoparticle and washed with DW and $\mathrm{MeOH}$ to remove the unreacted mixture. The product was dispersed in $5 \mathrm{~mL}$ of $\mathrm{MeOH}$ with addition of concentrated $\mathrm{HCl}(50 \mu \mathrm{L})$ and refluxed for overnight. Suspension was centrifuged (13,000 rpm, $30 \mathrm{~min}$ ) and the pellet washed by PBS and $\mathrm{MeOH}$ for several times and dried in vacuo. The surface amine group was quantified by fluorescamine assay. The loaded Ce6 was quantified by the UV-vis absorbance $(664 \mathrm{~nm})$ in DMSO to minimize the scattering of silica.

\section{Synthesis of singlet oxygen sensitive linker (SOSL)}

SOSL was prepared by our previous report. ${ }^{3}$ Briefly, 3-MPA (1.7 mL, $19.57 \mathrm{mmol})$ was activated by $30 \mathrm{wt} \% \mathrm{NaOMe}$ in $\mathrm{MeOH}(7.34 \mathrm{~mL}, 39.14 \mathrm{mmol})$ at 0 ${ }^{\circ} \mathrm{C}$ and the disodium salt of 3-MPA was dried in vacuo. The solution of cis-1,2-dichloroethylene $(781 \mu \mathrm{L}, 10.3$ $\mathrm{mmol})$ in $\mathrm{EtOH}(500 \mu \mathrm{L})$ was added into the 3-MPA suspension in DMF $(10 \mathrm{~mL})$ by dropping and the mixture was stirred at RT for $18 \mathrm{~h}$. The solution was diluted with water $(50 \mathrm{~mL})$ and $\mathrm{pH}$ was adjusted to 3 by $1 \mathrm{M} \mathrm{KHSO}_{4}$. The product was extracted by EtOAc $(3 \times 100 \mathrm{~mL})$ and the combined organic layers were washed with water $(2 \times 100 \mathrm{~mL})$ and brine $(100 \mathrm{~mL})$, dried with dry $\mathrm{Na}_{2} \mathrm{SO}_{4}$ and concentrated. Crude product was washed with diethyl ether to obtain the white powder and recrystallized with EtOAc / Hexane and dried under vacuum. (Yield $=64 \%$ )

\section{Preparation of SOSL-conjugated CeAP (CeAP-L) or singlet oxygen non-sensitive CeAP (CeAP-SA)}

Singlet oxygen sensitive linker (SOSL) was conjugated on the surface of CeAP by amide coupling reaction. The CeAP ( $57 \mathrm{mg}, 7.31 \mu \mathrm{mol}$ of amine) was dispersed in DMSO $(1 \mathrm{~mL})$ by sonication. SOSL $(8.6$ $\mathrm{mg}$, $5 \mathrm{eq}$ of amine), EDC- $\mathrm{HCl}(35 \mathrm{mg}, 25 \mathrm{eq})$ and NHS (21 mg, $25 \mathrm{eq}$ ) were dissolved in $4 \mathrm{~mL}$ of DMSO and stirred for $30 \mathrm{~min}$. CeAP suspension was added into the reaction mixture and stirred at dark for overnight. Final product was obtained by centrifugation $(13,000$ 
rpm, 10 min), washed with DMSO, DW and $\mathrm{MeOH}$ and dried in vacuo. Conjugated amount of SOSL was back-titrated through the quantification of remained amine by fluorescamine assay.

For control experiment, SOSL was altered by succinic anhydride (SA). ${ }^{4}$ Briefly, $10 \mathrm{mg}$ CeAP was dispersed in dry DMF ( $5 \mathrm{~mL})$ and SA $(3.7 \mathrm{mg}, 5 \mathrm{eq}$ to amine) as $1 \mathrm{~mL}$ of DMF suspension was added. The reaction mixture was stirred at dark for overnight and the final product was obtained by centrifugation (13,000 rpm, $10 \mathrm{~min})$. Obtained CeAP-SA was washed with DMF, DW and $\mathrm{MeOH}$ and dried in vacuo. Conjugated amount of SA was back-titrated through the quantification of remained amine by fluorescamine assay.

\section{Preparation of allyl-conjugated CeAP-L (CeAP-L-M)}

Allyl group was introduced on the surface of CeAP for further radical polymerization. ${ }^{5}$ Briefly, 19.5 $\mathrm{mg}$ of CeAP-L $(1.90 \mu \mathrm{mol}$ of $\mathrm{COOH}), 18 \mathrm{mg}$ of EDC- $\mathrm{HCl}(50 \mathrm{eq})$ and $11 \mathrm{mg}$ of NHS (50 eq) were dispersed in $2 \mathrm{~mL}$ of DMSO. $3 \mu \mathrm{L}$ of allylamine (20 eq) was then introduced after $30 \mathrm{~min}$ of stirring and further stirred at dark for overnight. Final product was obtained by centrifugation $(13,000 \mathrm{rpm}, 10 \mathrm{~min})$ and washed with DMSO, DW and MeOH. CeAP-SA was applied for control experiment in a same manner and CeAP-SA-M was obtained.

\section{Preparation of ${ }^{1} \mathrm{O}_{2}$-sensitive PEG-coated CeAP (CeAP-L-PEG)}

Singlet oxygen sensitive PEG was coated on the CeAP by polymerization. ${ }^{5}$ CeAP-L-M was uniformly dispersed in DW as $2 \mathrm{mg} / \mathrm{mL}$ suspension and $1 \mathrm{M}$ PEGDA $(9.6 \mu \mathrm{L}), 0.1 \mathrm{M}$ APS $(20 \mu \mathrm{L})$ and TEMED $(16$ $\mu \mathrm{L})$ was added. The reaction mixture was stirred at dark for overnight and CeAP-L-PEG was obtained by centrifugation. CeAP-SA-M was utilized for control experiment and CeAP-SA-PEG was obtained in a same manner.

\section{Loading doxorubicin in CeAP-L-PEG (DOX@CeAP-L-PEG)}

Doxorubicin (DOX), a typical anticancer drug, was loaded in CeAP-L-PEG by diffusion. Briefly, 10 $\mathrm{mg}$ of CeAP-L-PEG was dispersed in $10 \mathrm{~mL}$ of 1 $\mathrm{mg} / \mathrm{mL}$ DOX solution in DW and stirred at dark for overnight. Final product was obtained by centrifugation $(13,000 \mathrm{rpm}, 10 \mathrm{~min})$ and washed with DW. For quantification of loaded DOX, final product was dispersed in $\mathrm{MeOH}$ and fluorescence of extracted DOX (ex $=495 \mathrm{~nm} / \mathrm{em}=555 \mathrm{~nm}$ in $\mathrm{MeOH})$ was measured. DOX was loaded in CeAP-SA-PEG for control experiment.

\section{Detection of singlet oxygen}

Generation of singlet oxygen from CeAP and free Ce6 was measured by the fluorescence at $530 \mathrm{~nm}$ from the reacted product of SOSG and singlet oxygen. ${ }^{6}$ CeAP was dispersed in PBS to final concentration of $0.1 \mathrm{mg} / \mathrm{mL}$ or same equivalence of Ce6 $(2.3 \mu \mathrm{M})$ was dispersed and SOSG was added into the suspension to final concentration of $10 \mu \mathrm{M}$. Suspension was irradiated by red laser $(660 \mathrm{~nm}$ diode laser, Shanghai dream lasers, China) with final intensity of $100 \mathrm{~mW} / \mathrm{cm}^{2}$. Fluorescence of reacted SOSG (ex $=504 \mathrm{~nm} / \mathrm{em}=528 \mathrm{~nm})$ was measured at different time scales $(0,10,20,30,45,60 \mathrm{~min})$. Control experiment was operated by incubation in a dark condition.

\section{Photo-responsive release of DOX from DOX@CeAP-L-PEG}

In order to evaluate the photo-responsive drug release from DOX@CeAP-L-PEG, $0.1 \mathrm{mg} / \mathrm{mL}$ suspension of DOX@CeAP-L-PEG in buffer (PBS for $\mathrm{pH}=7.4$; acetate buffer for $\mathrm{pH}=5.0$ ) was irradiated by a red laser $\left(660 \mathrm{~nm}, 150 \mathrm{~mW} / \mathrm{cm}^{2}\right) .200 \mu \mathrm{L}$ aliquot of the suspension was collected at predetermined time intervals and centrifuged to measure the fluorescence of DOX (ex = $495 \mathrm{~nm} / \mathrm{em}=555 \mathrm{~nm})$ from the supernatant. A similar suspension incubated in absence of any light was used as a negative control. For control experiment, DOX@CeAP-SA-PEG was assessed by the same procedure.

\section{Cell viability test}

Nonspecific cytotoxicity of laser itself and the dose-dependent cytotoxicity of free DOX was measured. Cells (8000 cells/well; HeLa for laser toxicity; HeLa, PC-3, Hep3B and HCT-8 for DOX toxicity) were seeded on 96 well culture plates and incubated for overnight. For the nonspecific toxicity of irradiation, cells were irradiated by red laser $(660 \mathrm{~nm}$, diode laser) under fresh medium for $0,3,6,9,12$ or 15 min and further incubated for $24 \mathrm{~h}$. For free DOX toxicity, medium was replaced by fresh medium containing 0 to $20 \mu \mathrm{M}$ of DOX (HeLa and PC-3) or 0 to $50 \mu \mathrm{M}$ (Hep3B and HCT-8) and further incubated for $24 \mathrm{~h}$. After incubation, cell viability was evaluated by MTT assay.

For MTT assay, medium was replaced by $180 \mu \mathrm{L}$ of fresh medium and $20 \mu \mathrm{L}$ of MTT solution (5 $\mathrm{mg} / \mathrm{mL}$ ) was added. After incubation at dark for $4 \mathrm{~h}$, medium was removed and purple formazan crystal was completely dissolved by $200 \mu \mathrm{L}$ of DMSO. $100 \mu \mathrm{L}$ of each solution was transferred into a 96 well plate and UV-vis absorbance at $570 \mathrm{~nm}$ was measured by a microplate spectrofluorometer (VICTOR3 V multilabel counter). The relative percentages of 
non-treated cells were used to represent $100 \%$ of cell viability.

\section{Photo-triggered cytotoxicity in vitro}

The dose-dependent cytotoxicity of free Ce6 with and without irradiation was evaluated. Cells (8000 cells/well, HeLa, PC-3, Hep3B and HCT-8) were seeded on 96 well culture plates and incubated for overnight. Medium was replaced by fresh serum-free medium containing 0 to $500 \mathrm{ng} / \mathrm{mL}$ of Ce6 and incubated for $4 \mathrm{~h}$. After incubation, cells were washed with PBS and medium was replaced to fresh medium. Cells were then irradiated by red laser $(660 \mathrm{~nm}$ diode laser, Shanghai dream lasers, China) with power density of $150 \mathrm{~mW} / \mathrm{cm}^{2}$ for $15 \mathrm{~min}$ or incubated at dark and cytotoxicity was measured by MTT assay after further incubation for $24 \mathrm{~h}$.

Cytotoxicity of photo-responsive nanoparticles with and without irradiation were evaluated under various concentration. Cells (8000 cells/well, HeLa, PC-3, Hep3B and HCT-8) were seeded on 96 well culture plates and incubated for overnight. DOX@CeAP-L-PEG at final concentration of 0 to 20 $\mu \mathrm{g} / \mathrm{mL}$ CeAP-L-PEG at final concentration of same Ce6 equivalency compared to DOX@CeAP-L-PEG was treated under serum-free medium and incubated for $4 \mathrm{~h}$. Cells were then washed with PBS and irradiated by laser with power density of 150 $\mathrm{mW} / \mathrm{cm}^{2}$ for $15 \mathrm{~min}$ or incubated at dark under fresh medium and further incubated for $24 \mathrm{~h}$, followed by MTT assay. For control experiment, non-responsive nanoparticles (DOX@CeAP-SA-PEG or CeAP-SA-PEG) with and without irradiation was evaluated. Photo-toxicity at final concentration of same equivalency of Ce6 against HeLa cell was evaluated in a same manner.

\section{Intracellular release of DOX observed by confocal laser scanning microscopy (CSLM)}

Intracellular releasing property of DOX facilitated by red laser irradiation was analysed by confocal microscopy image. Briefly, HeLa cells (50,000 cells/well) were seeded on the glass cover slips placed in a 12 well culture plate and incubated for overnight. Medium was replaced by fresh serum-free medium containing $10 \mu \mathrm{g} / \mathrm{mL}$ DOX@CeAP-L-PEG or DOX@CeAP-SA-PEG. After $4 \mathrm{~h}$ of incubation, cells were washed carefully and irradiated by red laser $\left(150 \mathrm{~mW} / \mathrm{cm}^{2}\right)$ for $10 \mathrm{~min}$ under fresh medium. Cells were washed with PBS and fixed immediately with 10 $\%$ neutrally buffered formalin (NBF) just after irradiation or after $4 \mathrm{~h}$ of further incubation. Cells on the coverslip were mounted by Vectashield anti-fade mounting medium including DAPI (Vector Labs). Intracellular fluorescence of DOX was observed by
CLSM with excitation at $470 \mathrm{~nm}$ and emission at 556 $\mathrm{nm}$ and false-imaged as red. ${ }^{7}$

\section{Photochemical internalization (PCI) based endosomal escape monitored by CLSM}

In order to prove the photodynamically triggered endosomal escape, the localization of the carrier (CeAP-L-PEG) and the endo-lysosome were observed by confocal microscope. Briefly, HeLa cell (20,000 cells/well) was seeded on the glass cover slips placed in a 12 well culture plate and incubated for overnight. Then, medium was replaced by fresh serum-free medium containing $5 \mu \mathrm{g} / \mathrm{mL}$ CeAP-L-PEG and incubated for $4 \mathrm{~h}$. Cells were irradiated by $660 \mathrm{~nm}$ laser $\left(150 \mathrm{~mW} / \mathrm{cm}^{2}\right)$ for $10 \mathrm{~min}$ and lysotracker was added immediately at the final concentration of $4 \mu \mathrm{M}$. After $5 \mathrm{~min}$ of incubation, cellular uptake was blocked by cold DPBS and cells were washed thoroughly, finally fixed with $10 \%$ neutrally buffered formalin (NBF) for overnight at 4 ${ }^{\circ} \mathrm{C}$. Cells on the coverslip were mounted in Vectashield anti-fade mounting medium including DAPI (Vector Labs) and observed with CLSM. Fluorescence of lysotracker was false-imaged as green and Ce6 from CeAP-L-PEG was pseudo-coloured as red.

\section{In vivo experiment}

All animal experiments were approved by the Postech Biotech Center Ethics Committee. Cells (1 x $10^{6}$ CT26 cells) were inoculated subcutaneously (s.c.) into the flank of each female Balb/c mice weighing 17 $\pm 2 \mathrm{~g}$. After the average tumor volume reached 100 $\mathrm{mm}^{2}$, the mice were randomly divided into six groups (five mice per group) and treated with $100 \mu \mathrm{L}$ of physiological saline, free DOX, free Ce6, CeAP-L-PEG, DOX@CeAP-SA-PEG and DOX@CeAP-L-PEG $(2 \mathrm{mg} / \mathrm{kg}$ DOX and $83 \mu \mathrm{g} / \mathrm{kg}$ Ce6), intratumorally (i.t.). After $6 \mathrm{~h}$ of sample injection, mice were irradiated by the red laser (660 $\left.\mathrm{nm}, 150 \mathrm{~mW} / \mathrm{cm}^{2}, 20 \mathrm{~min}\right)$. The antitumor effect against CT26 cell was evaluated by measuring tumor volume. Each tumor volume was calculated from two dimensions measured by electronic calliper at desired time interval, by formula for a prolate ellipsoid; tumor volume was calculated as $a b^{2} / 2$ where $a$ is the longest and $b$ is the shortest dimension. The tumor growth was monitored until the 15 days after sample injection, at the time when mice were sacrificed. In order to demonstrate the statistical differences, Student's t-test was performed $\left({ }^{* *} p<0.01\right.$ and ${ }^{* *} p<$ 0.001). Furthermore, the tumor tissue of one mouse per group sacrificed at day 15 were collected for histological evaluation. 

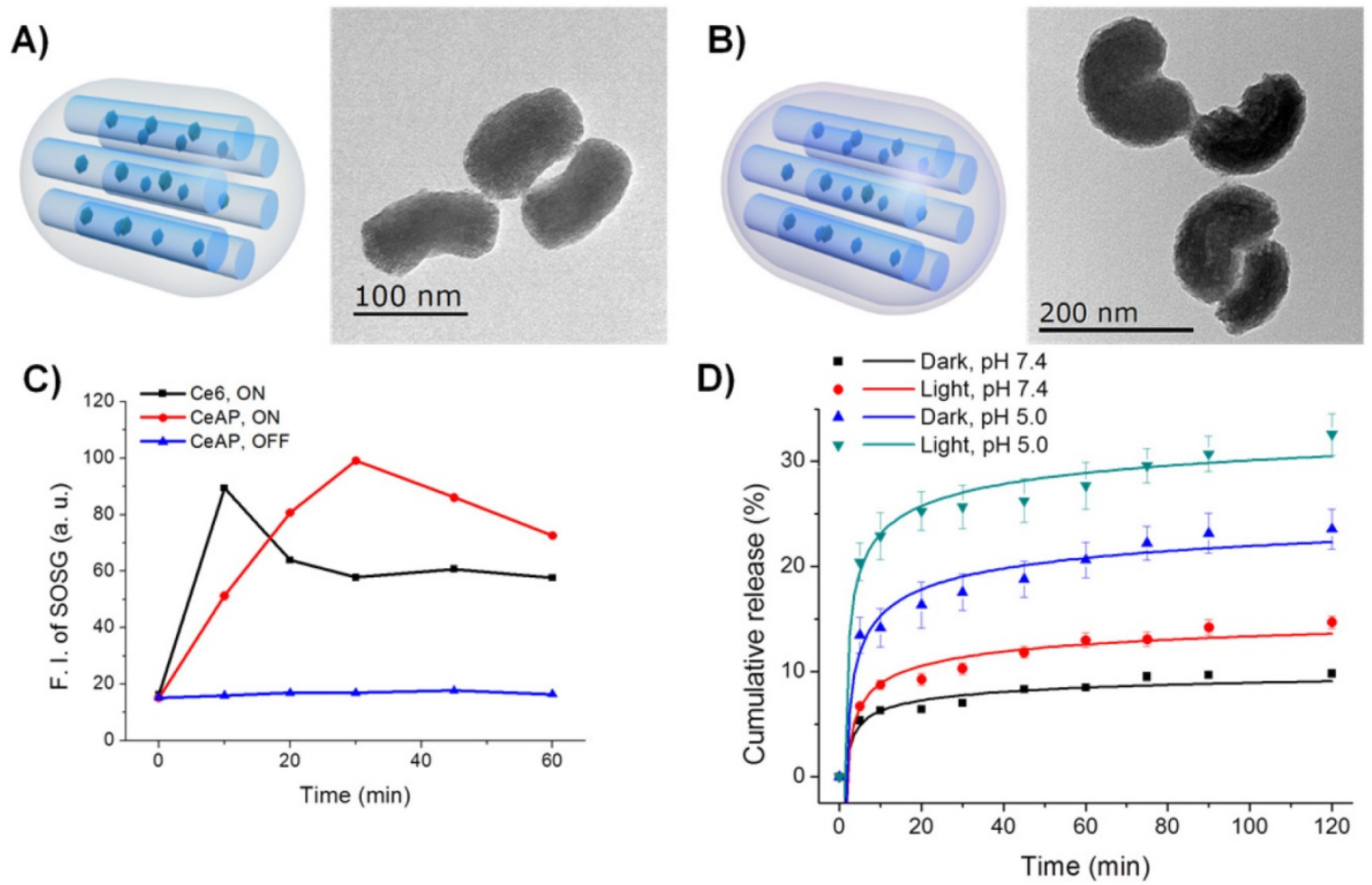

Figure 1. Illustration and TEM images of A) CeAP and B) CeAP-L-PEG. C) Photo-triggered generation of singlet oxygen determined by fluorescence of SOSG. D) Photo-responsive drug release of DOX@CeAP-L-PEG in vitro.

\section{Results and discussion}

\section{Synthesis and characterization of the drug carrier (DOX@CeAP-L-PEG)}

In order to introduce the red light-responsive PS in the channel of the MSNs, Ce6-conjugated organosilane (Ce6-TES) was synthesized by coupling the carboxylic acid of $\mathrm{Ce} 6$ with the amine-functionalized silane 3-aminopropyltriethoxysilane (APTES) through EDC-NHS chemistry. ${ }^{18}$ As-prepared Ce6-TES was incorporated during the synthesis of surface-amine-modified MSNs (CeAP), synthesized by following a previous report with some modifications. ${ }^{30}$ TEM imaging showed that the MSNs showed a kidney-like shape (longitudinal $\sim 160 \mathrm{~nm}$, transverse $\sim 80 \mathrm{~nm}$ ), which may be the result of hydrophobic interactions between Ce6-TES and $\mathrm{CTAB}$, corresponding to the results of a previous report (Figs. 1A, S1A). ${ }^{31}$ The UV-vis spectra of Ce6, Ce6-TES, and CeAP showed the inherent absorption of the Soret- and Q-band of the Ce6 molecule, indicating the non-aggregated nature of $\mathrm{Ce} 6$ molecules (Figs. S1B-C). In addition, the amount of Ce6 in CeAP was quantified by UV-vis absorption and it was calculated to be $13.6 \mathrm{mg}$ per $\mathrm{g}$ of nanoparticles.

Since Ce6 is chemically confined in the channel structure of CeAP, stable generation of ${ }^{1} \mathrm{O}_{2}$ should be confirmed to achieve photodynamically triggered drug release. Photosensitized generation of ${ }^{1} \mathrm{O}_{2}$ was monitored by the fluorescence of singlet oxygen sensor green (SOSG) under irradiation with a red laser $(660 \mathrm{~nm}){ }^{32}$ As shown in Fig. 1C, free Ce6 and CeAP exhibited similar maximum intensities of SOSG fluorescence, which reveals the preserved activity of the Ce6 molecule in CeAP. Interestingly, there was a difference between the time required to reach the maximum intensity of ${ }^{1} \mathrm{O}_{2}$ (10 min for free Ce6; $30 \mathrm{~min}$ for (eAP) and it is thought to be a result of the different accessibilities of triplet oxygen between Ce6 in the solution state and in the silica channel as a confined structure. This result implies the potential of $\mathrm{CeAP}$ as a template for the photodynamically assisted drug releasing system.

For the next step, the SOSL was synthesized and conjugated on the surface of CeAP to introduce ${ }^{1} \mathrm{O}_{2}$ sensitivity, following our previous report, and CeAP-L was obtained. ${ }^{28}$ The synthesis of SOSL was confirmed by ${ }^{1} \mathrm{H}$ NMR and Fourier transform infrared (FT-IR) spectroscopy (Fig. S2). The conjugated amount of linker was determined through an indirect quantification of residual primary amine by fluorescamine assay. ${ }^{33}$ The amount of $-\mathrm{NH}_{2}$ moiety on $\mathrm{CeAP}$ and $-\mathrm{COOH}$ moiety on CeAP-L were calculated to be 128 and $97.7 \mu \mathrm{mol}$ per $\mathrm{g}$ of nanoparticles, respectively. Subsequently, allylamine was 
conjugated by EDC-NHS chemistry for further polymerization, and PEG was conjugated by radical polymerization between allylamine and poly(ethylene glycol) diacrylate to obtain the nanoparticle CeAP-L-PEG. Successful functionalization of each modification step was revealed by the zeta potentials corresponding to amine (CeAP), carboxylic acid (CeAP-L), allylamine (CeAP-L-M), and PEG (CeAP-L-PEG) on the surface (Fig. S1D). The formation of the polymeric shell on the surface of CeAP was also confirmed by TEM imaging (Fig. 1B). In order to set up an ${ }^{1} \mathrm{O}_{2}$-insensitive negative control, the SOSL was altered with a succinic anhydride (SA) as an ${ }^{1} \mathrm{O}_{2}$-insensitive linker to introduce the PEG shell on the surface of CeAP. The entire synthetic procedure was performed in the same manner to obtain CeAP-SA-PEG.

Following the synthesis of the carrier (CeAP-L-PEG), a typical anti-cancer drug, doxorubicin (DOX), was loaded in the PEG shell and the porous structure of the MSNs to obtain DOX@CeAP-L-PEG. The amount of loaded DOX was confirmed by fluorescence after extraction in methanol. The calculated quantity of loaded DOX was $0.64 \mathrm{mg} / \mathrm{g}$ for DOX@CeAP-L-PEG and $0.60 \mathrm{mg} / \mathrm{g}$ for DOX@CeAP-SA-PEG, respectively.

\section{Photo-responsive drug release}

To evaluate the photodynamically assisted drug release property, in vitro release profiles of DOX@CeAP-L-PEG and DOX@CeAP-SA-PEG were monitored in solution at different $\mathrm{pH}(\mathrm{pH} 7.4$ and 5.0) with or without irradiation of red light $(660 \mathrm{~nm}, 150$ $\mathrm{mW} / \mathrm{cm}^{2}$ ). As shown in Fig. 1D and Fig. S3, both systems exhibited pH-responsive drug release behavior. The accelerated release at acidic $\mathrm{pH}$ can be explained by the inherent characteristics of the loaded DOX. At acidic $\mathrm{pH}$, the solubility of DOX could be increased by the protonation of the primary amine and drug release was enhanced by the reduced interaction between DOX and silica or the PEG shell. ${ }^{34}$ The $\mathrm{pH}$-induced drug release is a plausible behavior for successful intracellular drug release because during maturation, endolysosomal $\mathrm{pH}$ becomes acidic in comparison with physiological conditions. ${ }^{35}$ Besides the $\mathrm{pH}$-responsive profile, photo-responsive drug release was only observed from the drug carrier when the SOSL was introduced (Fig. 1D). Significant acceleration of drug release under irradiation with red light was observed from DOX@CeAP-L-PEG, which was driven by the generation of ${ }^{1} \mathrm{O}_{2}$ and further loosened linkage between silica and the PEG shell. In contrast, only a negligible difference was observed from the release profile of ${ }^{1} \mathrm{O}_{2}$-insensitive DOX@CeAP-SA-PEG regardless of external stimulus. These results clearly indicate the potential of CeAP-L-PEG as a photodynamically assisted drug release platform.

In addition to the solution level, it is important to exhibit stimuli-responsive drug release at the cellular level to show effective anti-cancer behavior. To that end, photo-responsive intracellular release of DOX was visualized by confocal laser scanning microscopy. HeLa cells were treated with either DOX@CeAP-L-PEG or DOX@CeAP-SA-PEG, irradiated by red light $\left(150 \mathrm{~mW} / \mathrm{cm}^{2}\right)$ for $10 \mathrm{~min}$, and observed after $4 \mathrm{~h}$. As demonstrated in Fig. 2, only the irradiated DOX@CeAP-L-PEG showed strong fluorescence signal from the nucleus region, indicating successful release of DOX and localization into the nucleus, where DOX works as an anti-cancer drug. Meanwhile, all other samples showed DOX signal mainly in the cytosolic regions, which could indicate insufficient release of DOX from the carrier. ${ }^{21}$ This result implies that DOX@CeAP-L-PEG exhibits stimuli-responsive drug release behavior not only in solution but also at the cellular level.

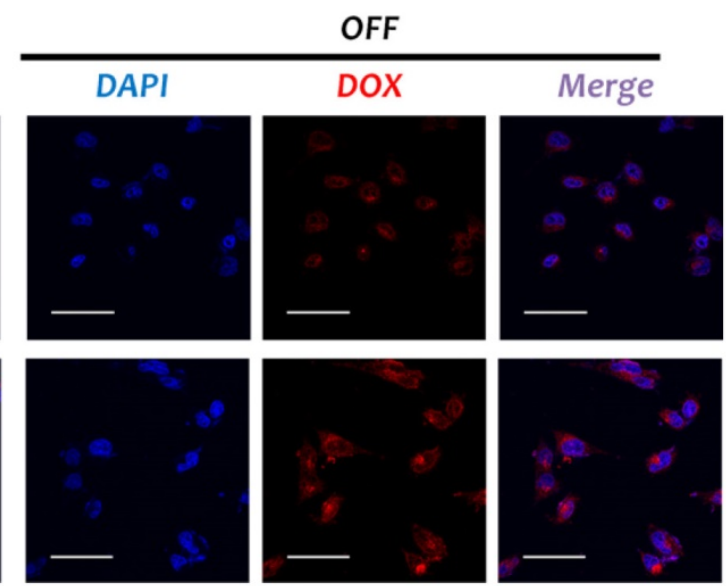

Figure 2. Confocal microscopic images of HeLa cells treated with DOX@CeAP-L-PEG (Linker) or DOX@CeAP-SA-PEG (Control) with or without irradiation of $660 \mathrm{~nm}$ laser $\left(150 \mathrm{~mW} / \mathrm{cm}^{2}\right)$. Nucleus was stained by DAPI (blue) and DOX was false-imaged as red. (scale bar $\left.=50 \mu \mathrm{m}\right)$ 


\section{Photo-responsive cytotoxicity and photochemical internalization}

As aforementioned, a single irradiation with red light can facilitate the generation of ${ }^{1} \mathrm{O}_{2}$ and drug release from DOX@CeAP-L-PEG in a sequence. As both photodynamic therapy and chemotherapy were expected, the photo-responsive therapeutic effect of DOX@CeAP-L-PEG was evaluated in vitro by MTT assay. To work as a stimuli-responsive drug delivery system, it is crucial that the cytotoxicity of the drug carrier should be induced only when irradiated by specific light. The stimulus condition used in this study, irradiation with red light $(660 \mathrm{~nm}$ laser, 150 $\mathrm{mW} / \mathrm{cm}^{2}$, maximum $\left.15 \mathrm{~min}\right)$, did not induce any significant toxicity in vitro (Fig. S4). In the dark, only negligible cytotoxicity was shown when the cells were treated nanoparticles, as expected, regardless of the loaded DOX (Figs. 3 and S6). Similar to the release pattern in Fig. S3, the ${ }^{1} \mathrm{O}_{2}$-insensitive system did not exhibit any notable difference in cytotoxicity regardless of the loaded drug, which reveals the insufficient release of the loaded drug under irradiation. In the case of CeAP-L-PEG, the ${ }^{1} \mathrm{O}_{2}$-sensitive nanoparticle without loaded DOX caused slightly decreased viability under irradiation, which was possibly due to the photodynamic effect from the photo-activated $\mathrm{Ce} 6$ in the MSN core. Moreover, DOX@CeAP-L-PEG showed the highest toxicity under irradiation with red light, represented by approximately $40 \%$ viability at a particle concentration of $20 \mu \mathrm{g} / \mathrm{mL}$ in the HeLa and PC-3 cell lines. In general, hepatocellular carcinoma and HCT-8 cell lines are known to be resistant to conventional chemotherapy including DOX. ${ }^{36-38}$ Interestingly, the equivalent $\mathrm{IC}_{50}$ values of DOX against Hep3B (12.7 $\mu \mathrm{M})$ and HCT-8 $(21.6 \mu \mathrm{M})$ were significantly reduced in comparison with those of free DOX, which were $40.3 \mu \mathrm{M}$ for Hep3B and 38.7 $\mu \mathrm{M}$ for HCT-8 (Fig. S6B). These results demonstrate that DOX@CeAP-L-PEG could be a practical alternative as a form of therapy for chemo-resistant cancer.
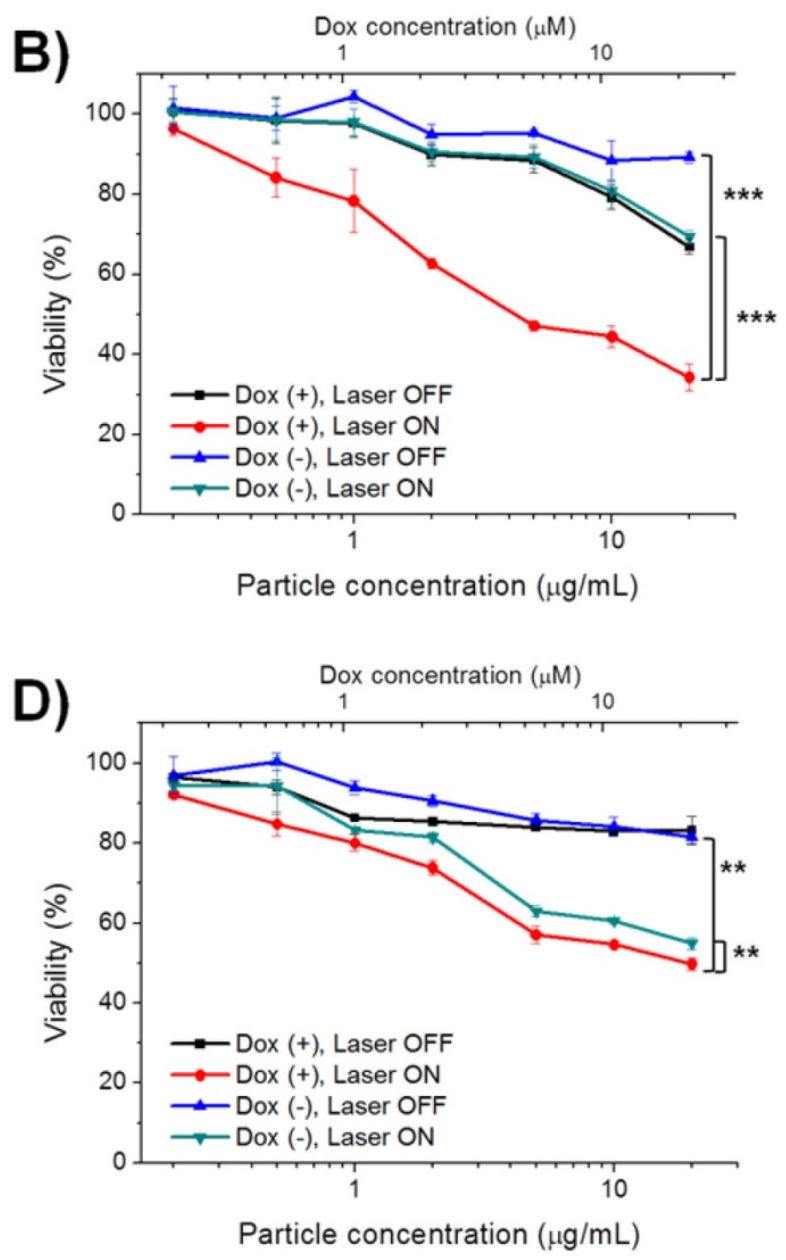

Figure 3. Photo-induced cytotoxicity of DOX@CeAP-L-PEG studied in A) HeLa, B) PC-3, C) Hep3B and D) HCT-8 cell line. (** $p<0.01, * * * p<0.001)$ 
In general, a nanoparticle-based drug delivery carrier should be allowed to enter cells and release the cargo drug intracellularly. Cellular uptake of nanoparticles is mainly followed by endocytic mechanisms involving acidification and enzymatic degradation of the internalized drug, thus the immediate escape from endosome is a key factor to exhibit the effect of a drug. ${ }^{39-41}$ At the cellular level, it has previously been reported that the photodynamic effect can promote endosomal escape, a process known as photochemical internalization (PCI). Through the photo-activation of PS, ROS are actively generated and the endosomal membrane is oxidized and further disrupted, followed by better escape of incorporated nanoparticles (Fig. 4A). ${ }^{25},{ }^{26}$ In order to prove that higher cytosolic delivery occurred by the photo-irradiated Ce6-based carrier and subsequent better endosomal escape, the localization of an endosome and the carrier (CeAP-L-PEG) was observed by confocal microscopy (Fig. 4B). Without irradiation, the intracellular red signal from $\mathrm{Ce} 6$ of the carrier was overlapped by the green signal from the lysotracker-labeled endosome, which indicates the confinement of nanoparticles in the endosome. Otherwise, better endosomal escape of the carrier in the cell was observed after irradiation as represented by the red signal (white arrow), which did not overlap with the green signal. Taken together, an enhanced therapeutic effect was induced by a single irradiation with red light through these sequential steps: 1) uncapping of the PEG shell and facilitated release of DOX; 2) better endosomal escape of nanoparticles by the PCI mechanism; and 3) dual effect of photodynamic therapy and chemotherapy.
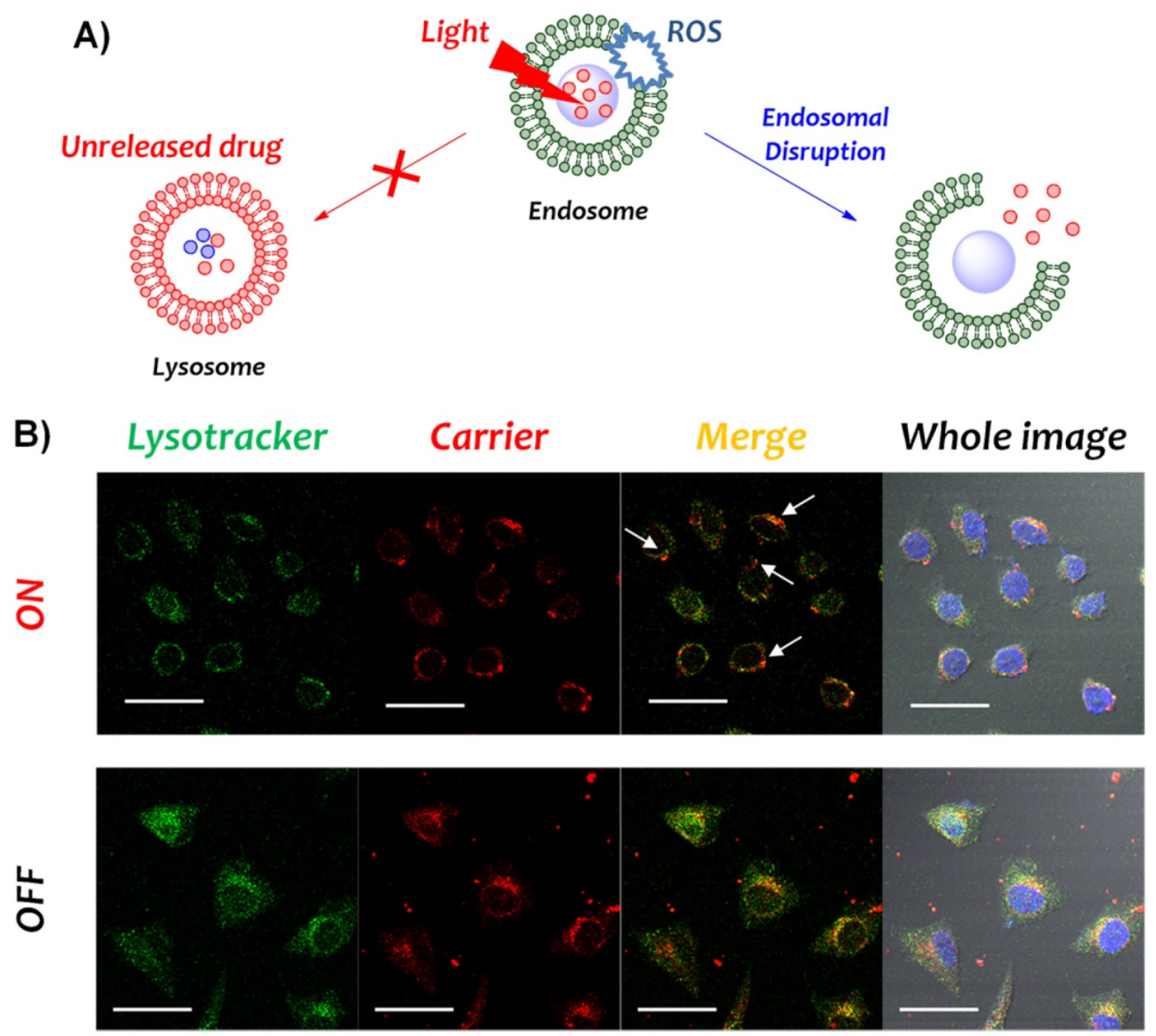

Figure 4. A) Schematic illustration of photochemical internalization (PCl). B) Confocal microscopic images of HeLa cells treated with CeAP-L-PEG with or without irradiation of $660 \mathrm{~nm}$ laser $\left(100 \mathrm{~mW} / \mathrm{cm}^{2}\right.$ ). Nucleus was stained by DAPI (blue), lysosome was stained by lysotracker (green) and the fluorescence of Ce6 from the carrier was false-imaged as red. (scale bar $=50 \mu \mathrm{m}$ ) 

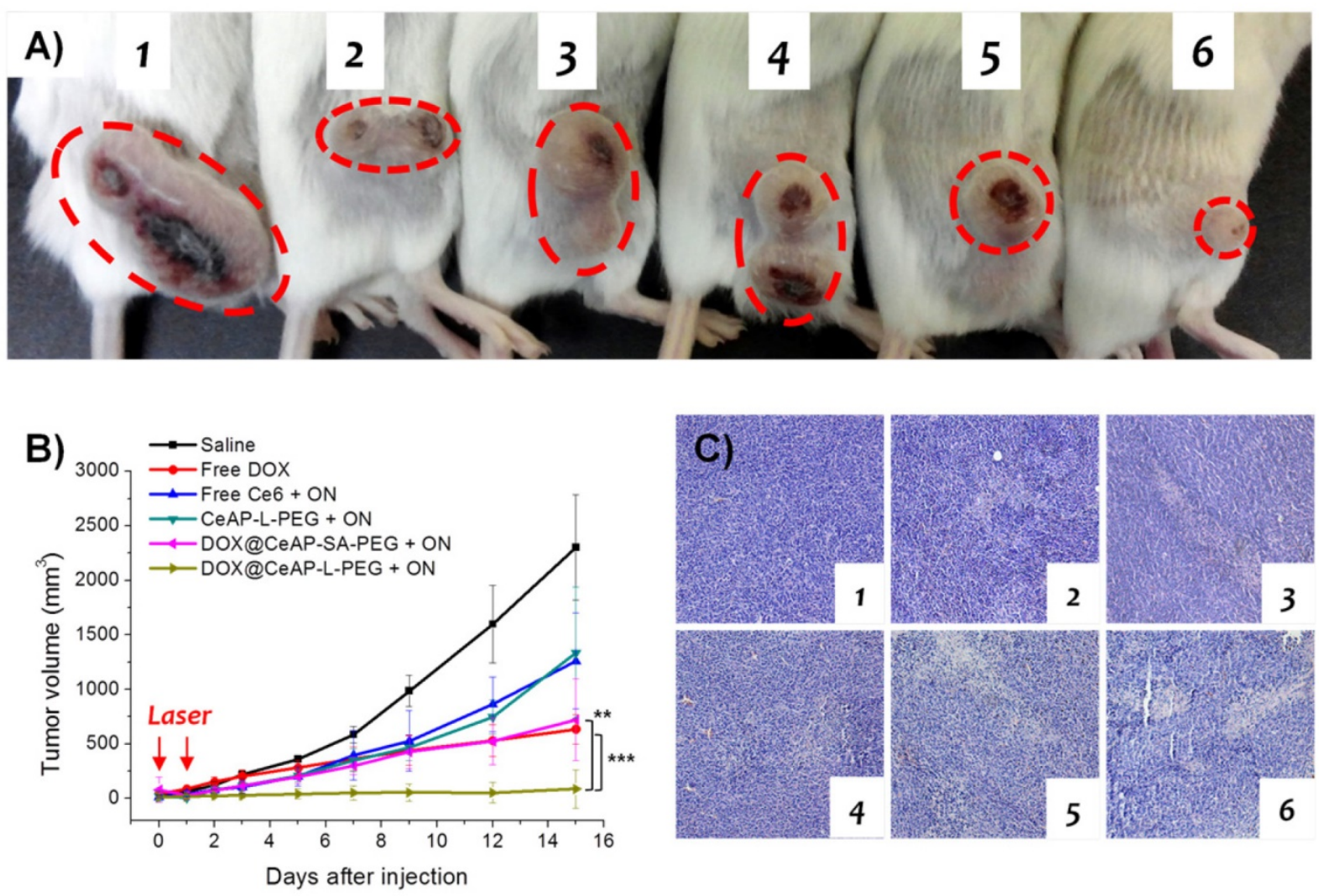

Figure 5. In vivo anti-tumor study. A) Representative image of CT-26 bearing balb/c mice. Each label represents; 1: saline; 2: Free DOX; 3: Free Ce6 + ON; 4: CeAP-L-PEG + ON; 5: DOX@CeAP-L-PEG + ON; 6: DOX@CeAP-L-PEG + ON. B) Tumor growth in CT-26 bearing balb/c mice $(n=5 ; * * p<0.01, * * * p<0.001)$. c) H\&E staining of tumor tissues. Representing numbers are same as 5 a.

\section{In vivo anti-cancer efficiency}

As observed above, an enhanced therapeutic effect of DOX@CeAP-L-PEG under irradiation with red light was confirmed in comparison with other control materials. The main strategy is mediated by as-generated ${ }^{1} \mathrm{O}_{2}$, followed by facilitated drug release and further escape from endosomes along with photodynamic therapy. In accordance with the enhanced therapeutic effect in vitro, photo-responsive anti-cancer effect is expected in vivo. To that end, CT26 colon cancer was selected as a tumor model owing to its fast and aggressive growth. Following the control samples used in vitro, 1) saline, 2) free DOX, 3) free Ce6, 4) CeAP-L-PEG, 5) DOX@CeAP-L-PEG, and 6) DOX@CeAP-SA-PEG were injected into CT26 tumor-bearing mice intratumorally in order to monitor the regression of tumor growth. For the photo-responsive samples, irradiation was done with a $660 \mathrm{~nm}$ red laser at days 0 and 1 . As demonstrated in Fig. 5A, CT26 actively grew for 15 days when only saline was injected. Free Ce6 and CeAP-L-PEG showed similar therapeutic effects, which might be caused by the photodynamic effect. As expected, chemotherapeutic effect was notably represented by free DOX. DOX@CeAP-SA-PEG, which is insensitive to light, showed partial therapeutic effect, which might be due to the photodynamic effect and some non-specific release of DOX from the carrier. Finally, the photo-responsive DOX@CeAP-L-PEG exhibited the most significant therapeutic effect, as noted in Fig. 5B, as a result of the dual effect of chemotherapy and photodynamic therapy observed in the in vitro results. Furthermore, the regression of tumor growth was verified at the tissue level by hematoxylin and eosin staining (Fig. 5C). The tumor treated with DOX@CeAP-L-PEG showed the highest level of apoptosis, resulting in the most regressed tumor growth. These results prove that photodynamically assisted drug release carriers, not only DOX@CeAP-L-PEG, are promising materials for stimuli-responsive therapeutic application.

\section{Conclusion}

Herein, a red light-responsive drug delivery system (DOX@CeAP-L-PEG) was prepared by the combination of a photosensitizer (Ce6) and the singlet oxygen-sensitive linker (SOSL). Succeeding our previous report, which led to the model study, the system was realized by the introduction of PEG on the shell for biological application, and the photodynamic and chemotherapeutic effects were evaluated. 
Photodynamically assisted drug release was controlled by external irradiation with biocompatible red light and enhanced therapeutic effect was confirmed in vitro and in vivo. Additionally, intracellular drug release and PCI-mediated endosomal escape of the carrier were successfully confirmed. With high biocompatibility and the photodynamically assisted release of loaded anti-cancer drugs, our system has a potential to offer the combination of photodynamic therapy and chemotherapy for the treatment of cancer.

\section{Supplementary Material}

Supplementary schemes and figures.

http://www.ntno.org/v01p0196s1.pdf

\section{Acknowledgement}

This work was supported by the research center program of IBS (Institute for Basic Science) in Korea (IBS-R007-G2).

\section{Competing Interests}

The authors have declared that no competing interest exists.

\section{References}

1. Gottesman MM, Fojo T, Bates SE. Multidrug resistance in cancer: role of ATP-dependent transporters. Nat Rev Cancer 2002; 2: 48-58.

2. Holohan C, Van Schaeybroeck S, Longley DB, Johnston PG. Cancer drug resistance: an evolving paradigm. Nat Rev Cancer 2013; 13: 714-26.

3. Timko BP, Dvir T, Kohane DS. Remotely triggerable drug delivery systems. Adv Mater. 2010; 22: 4925-43.

4. Lee J, Park H, Kim WJ. Nano "Chocolate Waffle" for near-IR Responsive Drug Releasing System. Small 2015; 11: 5315-23.

5. Lee J, Jeong C, Kim WJ. Facile fabrication and application of near-IR light-responsive drug release system based on gold nanorods and phase change material. J Mater Chem B 2014; 2: 8338-8345.

6. He C, Liu D, Lin W. Self-assembled core-shell nanoparticles for combined chemotherapy and photodynamic therapy of resistant head and neck cancers. ACS Nano 2015; 9: 991-1003.

7. Peterson CM, Lu JM, Sun Y, Peterson CA, Shiah JG, Straight RC, Kopecek J. Combination chemotherapy and photodynamic therapy with $\mathrm{N}$-(2-hydroxypropyl) methacrylamide copolymer-bound anticancer drugs inhibit human ovarian carcinoma heterotransplanted in nude mice. Cancer Res 1996; 56: 3980-5.

8. Luo D, Carter KA, Geng J, Shao S, Giraldo D, Sunar U, Ortega J, Fovell JF. Doxorubicin encapsulated in stealth liposomes conferred with light-triggered drug release. Biomaterials 2016; 75: 193-202.

9. Dougherty TJ. Photosensitizers: therapy and detection of malignant tumors. Photochem Photobiol. 1987; 45: 879-89.

10. Dolmans DE, Fukumura D, Jain RK. Photodynamic therapy for cancer. Nat Rev Cancer 2003; 3: 380-7.

11. DeRosa MC, Crutchley RJ. Photosensitized singlet oxygen and its applications. Coord Chem Rev. 2002; 233-234: 351-371.

12. Henderson BW, Busch TM, Vaughan LA, Frawley NP, Babich D, Sosa TA, Zollo JD, Dee AS, Cooper MT, Bellnier DA, Greco WR, Oseroff AR. Photofrin photodynamic therapy can significantly deplete or preserve oxygenation in human basal cell carcinomas during treatment, depending on fluence rate. Cancer Res 2000; 60: 525-9.

13. Bechet D, Couleaud P, Frochot C, Viriot ML, Guillemin F, Barberi-Heyob M. Nanoparticles as vehicles for delivery of photodynamic therapy agents. Trend Biotechnol. 2008; 26: 612-21.

14. Couleaud P, Morosini V, Frochot C, Richeter S, Raehm L, Durand JO. Silica-based nanoparticles for photodynamic therapy applications. Nanoscale 2010; 2: 1083-95.

15. Gary-Bobo M, Mir Y, Rouxel C, Brevet D, Basile I, Maynadier M, Vaillant $\mathrm{O}$, Mongin O, Blanchard-Desce M, Morere A, Garcia M, Durand JO, Raehm L. Mannose-functionalized mesoporous silica nanoparticles for efficient two-photon photodynamic therapy of solid tumors. Angew Chem Int Ed. 2011; 50: 11425-9.
16. Koo H, Lee $\mathrm{H}$, Lee $\mathrm{S}$, Min KH, Kim MS, Lee DS, Choi Y, Kwon IC, Kim K, Jeong SY. In vivo tumor diagnosis and photodynamic therapy via tumoral pH-responsive polymeric micelles. Chem Commun. 2010; 46: 5668-70.

17. Lee SJ, Koo H, Jeong H, Huh MS, Choi Y, Jeong SY, Byun Y, Choi K, Kim K, Kwon IC. Comparative study of photosensitizer loaded and conjugated glycol chitosan nanoparticles for cancer therapy. J Control Release 2011; 152: 21-9.

18. Ohulchanskyy TY, Roy I, Goswami LN, Chen Y, Bergey EJ, Pandey RK, Oseroff AR, Prasad PN. Organically modified silica nanoparticles with covalently incorporated photosensitizer for photodynamic therapy of cancer. Nano Lett. 2007; 7: 2835-42.

19. Peng CL, Lai PS, Lin FH, Yueh-Hsiu Wu S, Shieh MJ. Dual chemotherapy and photodynamic therapy in an HT-29 human colon cancer xenograft model using SN-38-loaded chlorin-core star block copolymer micelles. Biomaterials 2009; 30: 3614-25.

20. Qian HS, Guo HC, Ho PC, Mahendran R, Zhang Y. Mesoporous-silica-coated up-conversion fluorescent nanoparticles for photodynamic therapy. Small 2009; 5: 2285-90.

21. Saravanakumar G, Lee J, Kim J, Kim WJ. Visible light-induced singlet oxygen-mediated intracellular disassembly of polymeric micelles co-loaded with a photosensitizer and an anticancer drug for enhanced photodynamic therapy. Chem Commun. 2015; 51: 9995-8.

22. van Nostrum CF. Delivery of photosensitizers in photodynamic therapy. Adv Drug Deliv Rev. 2004; 56: 5-6.

23. Wieder ME, Hone DC, Cook MJ, Handsley MM, Gavrilovic J, Russell DA. Intracellular photodynamic therapy with photosensitizer-nanoparticle conjugates: cancer therapy using a 'Trojan horse'. Photochem Photobiol Sci. 2006; 5: 727-34.

24. Henderson BW, Dougherty TJ. Photodynamic therapy : basic principles and clinical applications. New York, USA: CRC Press; 1992.

25. Berg K, Selbo PK, Prasmickaite L, Tjelle TE, Sandvig K, Moan J, Gaudernack G, Fodstad O, Kjolsrud S, Anholt H, Rodal GH, Rodal SK, Hogset A. Photochemical internalization: a novel technology for delivery of macromolecules into cytosol. Cancer Res. 1999; 59: 1180-3.

26. Nishiyama N, Iriyama A, Jang WD, Miyata K, Itaka K, Inoue $Y$, Takahashi $H_{\text {, }}$ Yanagi $Y$, Tamaki $Y$, Koyama H, Kataoka K. Light-induced gene transfer from packaged DNA enveloped in a dendrimeric photosensitizer. Nat Mater. 2005; 4: 934-41.

27. Moses B, You Y. Emerging strategies for controlling drug release by using visible/near IR light. Med Chem. 2013; 3: 192-198.

28. Lee J, Park J, Singha K, Kim WJ. Mesoporous silica nanoparticle facilitated drug release through cascade photosensitizer activation and cleavage of singlet oxygen sensitive linker. Chem Commun. 2013; 49: 1545-7.

29. Singh N, Karambelkar A, Gu L, Lin K, Miller JS, Chen CS, Sailor MJ, Bhatia $\mathrm{SN}$. Bioresponsive mesoporous silica nanoparticles for triggered drug release. I Am Chem Soc. 2011; 133: 19582-5.

30. Slowing I, Trewyn BG, Lin VS. Effect of surface functionalization of MCM-41-type mesoporous silica nanoparticles on the endocytosis by human cancer cells. J Am Chem Soc. 2006; 128: 14792-3.

31. Ahn B, Park J, Singha K, Park H, Kim WJ. Mesoporous silica nanoparticle-based cisplatin prodrug delivery and anticancer effect under reductive cellular environment. J Mater Chem B 2013; 1: 2829-2836.

32. Gollmer, A, Arnbjerg, J, Blaikie, F. H, Pedersen, B. W, Breitenbach, T, Daasbjerg, K, Glasius, M, Ogilby, P. R. Singlet Oxygen Sensor Green(R): photochemical behavior in solution and in a mammalian cell. Photochem Photobiol. 2011; 87: 671-9.

33. Chen Y, Zhang Y. Fluorescent quantification of amino groups on silica nanoparticle surfaces. Anal Bioanal Chem. 2011; 399: 2503-9.

34. Zhang Z, Wang L, Wang J, Jiang X, Li X, Hu Z, Ji Y, Wu X, Chen C. Mesoporous silica-coated gold nanorods as a light-mediated multifunctional theranostic platform for cancer treatment. Adv Mater. 2012; 24: 1418-23.

35. Kim J, Lee YM, Kang Y, Kim WJ. Tumor-homing, size-tunable clustered nanoparticles for anticancer therapeutics. ACS Nano 2014; 8: 9358-67.

36. Xu Y, Xia F, Ma L, Shan J, Shen J, Yang Z, Liu J, Cui Y, Bian X, Bie P, Qian C. MicroRNA-122 sensitizes HCC cancer cells to adriamycin and vincristine through modulating expression of MDR and inducing cell cycle arrest. Cancer Lett. 2011; 310: 160-9.

37. Park JG, Lee SK, Hong IG, Kim HS, Lim KH, Choe KJ, Kim WH, Kim YI, Tsuruo T, Gottesman MM. MDR1 gene expression: its effect on drug resistance to doxorubicin in human hepatocellular carcinoma cell lines. I Natl Cancer Inst. 1994; 86: 700-5.

38. Park H, Park W, Na K. Doxorubicin loaded singlet-oxygen producible polymeric micelle based on chlorine e6 conjugated pluronic F127 for overcoming drug resistance in cancer. Biomaterials 2014; 35: 7963-9.

39. Kim J, Kim H, Kim WJ. Single-layered MoS2-PEI-PEG nanocomposite-mediated gene delivery controlled by photo and redox stimuli. Small 2016; 12: 1184-92.

40. Kim J, Kim J, Jeong C, Kim WJ. Synergistic nanomedicine by combined gene and photothermal therapy. Adv Drug Delev Rev. 2016; 98: 99-112.

41. Lee J, Kim J, Kim WJ. Photothermally controllable cytosolic drug delivery based on core-shell MoS2-porous silica nanoplates. Chem Mater. 2016; 28: 6417-24. 


\section{Author Biography}

Dr. Junseok Lee received his B.S. and Ph.D. from the Department of Chemistry of Pohang University of Science and Technology (POSTECH) in 2010 and 2015 respectively under the supervision of Prof. Won Jong Kim. Now he is a post-doctoral researcher of Center for Self-assembly and Complexity, Institute for Basic Science (IBS) in Korea since 2015. His main research interests are designing various functional nanoparticles for photo-responsive drug delivery system.

Dr. Yeong Mi Lee received her B.S., M.S., and Ph.D from the Department of Biomedical Science of Inje University in 2002, 2004, and 2007 respectively under the supervision of Prof. Chong-Rak Kim. She has worked in Ulsan University and Seoul National University and POSTECH as a post-doctoral researcher and research professor. Now she is a post-doctoral researcher of the Department of Chemistry, POSTECH in Prof. Won Jong Kim's group. Her main research interests concern the development of various biopolymers for gene and drug delivery applications.

Dr. Jinhwan Kim obtained his B.S. from the Department of Bioscience and Biotechnology of Konkuk University in 2012. He received his Ph.D. from the Department of Chemistry of POSTECH in 2017 under the supervision of Prof. Won Jong Kim. He is now a post-doctoral researcher of Center for Self-assembly and Complexity, IBS in Korea since 2017. He has focused on the stimuli-regulated functional gene/drug delivery carriers for overcoming various biological barriers.

Dr. Won Jong Kim received his B.S. from Hanyang University in 1998, and M.S. and Ph.D. in Biomolecular Engineering in 2004 at Tokyo Institute of Technology under the supervision of Profs. T. Akaike and A. Maruyama. From 2004 to 2007, he was a post-doctoral fellow at the Prof. Sung Wan Kim's group in University of Utah. Currently, he is an associate professor at the Department of Chemistry, POSTECH, and group leader of Center for Self-assembly and Complexity, IBS in Korea. 